\title{
Post-Operative Spinal Epidural Hematoma after Thoracic and Lumbar Spinous Process-Splitting Laminectomy for Thoracic and Lumbar Spinal Stenosis
}

\author{
Yawara Eguchi ${ }^{1)}$, Munetaka Suzuki ${ }^{1)}$, Takashi Sato ${ }^{2)}$, Hajime Yamanaka ${ }^{1)}$, Hiroshi Tamai ${ }^{1)}$, Tatsuya Kobayashi ${ }^{1)}$, \\ Sumihisa Orita ${ }^{2)}$, Miyako Suzuki ${ }^{2)}$, Kazuhide Inage ${ }^{2)}$, Hirohito Kanamoto ${ }^{2)}$, Koki Abe ${ }^{2)}$, Masaki Norimoto ${ }^{2)}$, \\ Tomotaka Umimura ${ }^{2)}$, Yasuchika Aoki ${ }^{3)}$, Masao Koda ${ }^{4)}$, Takeo Furuya ${ }^{2)}$, Junichi Nakamura ${ }^{2)}$, Tsutomu Akazawa ${ }^{5)}$, \\ Kazuhisa Takahashi ${ }^{2}$ and Seiji Ohtori ${ }^{2}$
}

1) Department of Orthopaedic Surgery, Shimoshizu National Hospital, Yotsukaido, Japan

2) Department of Orthopaedic Surgery, Graduate School of Medicine, Chiba University, Chiba, Japan

3) Department of Orthopaedic Surgery, Eastern Chiba Medical Center, Chiba, Japan

4) Department of Orthopaedic Surgery, University of Tsukuba, Tsukuba, Japan

5) Department of Orthopaedic Surgery, St. Marianna University School of Medicine, Kanagawa, Japan

\begin{abstract}
:
Introduction: To investigate the risk of epidural hematoma after spinous process-splitting laminectomy (SPSL).

Methods: A total of 137 cases (mean age, 72.4 years; 68 men) of SPSL were included. Of these, there were instances (3.7\%; mean age, 70.5 years; all male) of postoperative development of new neurologic deficit due to epidural hematoma requiring reoperation. The 133 subjects (72.5 years; $64 \mathrm{men}$ ) with normal postoperative course were used as controls, and comparisons were made between both groups using chi-squared and Student's $t$-tests. Regarding our investigation of risk factors for epidural hematoma, logistic regression was conducted with presence or absence of hematoma as our primary outcome variable, and age, gender, disease duration, number of laminectomies, which levels were decompressed, blood loss, length of case, drain output, coagulopathy, and whether or not there was an intraoperative dural tear were our explanatory variables.
\end{abstract}

Results: All cases of hematoma were single-level laminectomies; there was one case of T9-10 and 3 cases of L2-3. In our direct comparison of both groups (hematoma versus control), the proportion of men was significantly higher in the hematoma group $(100 \%$ versus $48 \%, \mathrm{p}<0.05)$; levels decompressed were also significantly higher $(\mathrm{p}<0.05)$ in the hematoma group, and drain outputs were significantly lower $(113 \mathrm{~mL}$ versus $234 \mathrm{~mL}, \mathrm{p}<0.05)$. From our logistic regression analysis, the levels were significantly higher $\left(\chi^{2}=15, \mathrm{p}=0.0001\right)$ and the drain outputs were smaller $\left(\chi^{2}=4.6, \mathrm{p}=0.03\right)$ in the hematoma group.

Conclusions: Single-level decompression higher than the L2-3 level and reduced drain output were risk factors for spinal epidural hematoma. With this method of spinous process suturing and reconstruction there is less decompression compared with more conventional methods; therefore, the effect of hematoma may be more pronounced at higher vertebral levels with reduced canal width, and drain failure may also occur with this limited space.

Keywords:

lumbar spinal stenosis, risk factors, spinal epidural hematoma, spinous process-splitting laminectomy

Spine Surg Relat Res 2019; 3(3): 244-248 dx.doi.org/10.22603/ssrr.2018-0086

\section{Introduction}

Lumbar spinal canal stenosis (LSS) has increased with the aging of the population in recent years. In a large-scale study of 938 patients with a mean age of 66 years, its prevalence was approximately $10 \%$. This condition impairs quality of life and has now become a major social issue ${ }^{1)}$.

Although laminectomy for LSS is the most common sur-

Corresponding author: Yawara Eguchi, yawara_eguchi@yahoo.co.jp

Received: October 16, 2018, Accepted: December 17, 2018, Advance Publication: January 25, 2019

Copyright (C) 2019 The Japanese Society for Spine Surgery and Related Research 
Table 1.

\begin{tabular}{|c|c|c|c|c|c|c|c|c|c|}
\hline No. & Age & Gender & $\begin{array}{c}\text { No. of } \\
\text { laminectomy }\end{array}$ & $\begin{array}{c}\text { Level of } \\
\text { decompression }\end{array}$ & $\begin{array}{c}\text { Drain } \\
\text { output }(\mathrm{ml})\end{array}$ & $\begin{array}{c}\text { Time of } \\
\text { onset (day) }\end{array}$ & $\begin{array}{c}\text { Urinary } \\
\text { disturbance }\end{array}$ & $\begin{array}{l}\text { MMT } \\
\text { (onset) }\end{array}$ & $\begin{array}{l}\text { MMT } \\
\text { (final) }\end{array}$ \\
\hline 1 & 74 & Male & 1 & $\mathrm{~L} 2 / 3$ & 200 & 8 & + & 5 & 5 \\
\hline 2 & 55 & Male & 1 & T9/10 & 2 & 3 & - & $3 \sim 4$ & 5 \\
\hline 3 & 72 & Male & 1 & $\mathrm{~L} 2 / 3$ & 27 & 2 & - & 3 & 5 \\
\hline 4 & 81 & Male & 1 & $\mathrm{~L} 2 / 3$ & 225 & 1 & + & $0 \sim 1$ & 3 \\
\hline
\end{tabular}

MMT: manual muscle test

gical technique, complications such as muscle atrophy and decreased lumbar strength due to extensive paraspinal muscle detachment, postoperative kyphosis, and intervertebral instability have been reported. Therefore, various techniques for lumbar spinal canal enlargement preserving the posterior supporting tissues have been devised including spinous process osteotomy ${ }^{2,3)}$, fenestration ${ }^{4}$, and unilateral laminotomy for bilateral decompression ${ }^{5)}$. Lumbar spinous processsplitting laminectomy (SPSL) is a minimally-invasive technique that can preserve the posterior supporting tissues including the paraspinal muscles and interspinous ligaments ${ }^{6}$.

Neurologic deficit due to epidural hematoma may occur as a complication following spine surgery. Nevertheless, there are few reports on the incidence of postoperative epidural hematoma following SPSL. Accordingly, the purpose of this study is to investigate the risk of epidural hematoma following SPSL.

\section{Materials and Methods}

Informed consent was obtained from all participants before the study began. The study protocol was approved by the ethical review committee.

A total of 137 cases (mean age, 72.4 years; 68 men) of lumbar SPSL conducted from April 2013 until November 2017 were included.

The patient exclusion criteria were as follows: 1) those who had previous lumbar spinal surgery and 2) those who had spinal tumors, infectious disease, or spinal trauma; 87 subjects underwent single-level decompression, 40 subjects underwent 2-level decompression, and 10 subjects underwent 3-level decompression. The levels decompressed were T9-10, T12-L1, and L1-2 once each, L2-3 for 23 cases, L34 for 26 cases, L4-5 for 97 cases, and L5-S1 for five cases.

Here, we describe a case of single-level (L4-5) decompression, according to the surgical method described by Watanabe et al. ${ }^{6}$ as follows: a posterior midline skin incision is made between the L4 and L5 spinous processes. Soft tissue is dissected up to the tip of the L4 and L5 spinous processes using an electric knife. The cortex at the tip of the L4 spinous processes is removed at the midline by approximately $2 \mathrm{~cm}$ using a 4-mm diamond burr and a chisel. The spinous process is divided at the base, detached from the lamina using a cob elevator, and retracted using TrimLine (Medtronic, Sofamor Danek, Memphis, TN, USA). The remaining spinous process is removed using Luer bone rongeur forceps. Then, laminectomy is performed. After decompression, a suction drain is placed in the epidural space. After that, the spinous process and interspinal ligament, which were split, are sutured using absorbable thread. The drain is removed on postoperative day 2 .

There were four instances (mean age, 70.5 years; all male) of postoperative development of new neurologic deficit due to epidural hematoma requiring reoperation. The 133 subjects (mean age, 72.5 years; 64 men) with normal postoperative course were used as controls, and comparisons were made between both groups using chi-squared and Student's $t$-tests. Regarding our investigation of risk factors for epidural hematoma, logistic regression was conducted with presence or absence of hematoma as our primary outcome variable, and age, gender, disease duration, number of laminectomies, which levels were decompressed, blood loss, time of surgery, drain output, coagulopathy, and whether or not there was an intraoperative dural tear were our explanatory variables. Regarding which levels were decompressed, we considered 1 point for levels L1-2 or higher, 2 points for L2-3, 3 points for L3-4, 4 points for L4-5, and 5 points for L5-S1. For cases where multiple levels were decompressed, scoring was based on the central decompressed level. For example, we defined 3.5 points for 2-level decompression of L3-4 and L4-5, and 3 points for 3-level decompression of L 2-3, L3-4, and L4-5. Statistical analyzes were performed with Stat View software (version 5.0, SAS institute, Cary, NC). All data are expressed as mean \pm standard deviation. A threshold of $\mathrm{p}<0.05$ was considered statistically significant.

\section{Results}

\section{Descriptive statistics for epidural hematoma patients}

Table 1 contains descriptive statistics for the four patients who experienced neurologic deficits due to postoperative epidural hematoma and required reoperation. The frequency of occurrence was $3.7 \%$ (4/137), all subjects were men, all were single-level decompressions, and the level of decompression was at L2-3 or higher for all (Table 1).

\section{Results of the statistical analysis}

For each item (SPSL group, control group, p-value), the results were as follows: age $(70.5,72.5, \mathrm{p}=0.672)$, gender (4 male [100\%], 64 male [48.2\%], $\mathrm{p}=0.041$ ), disease duration in months $(17.3,33.1, \mathrm{p}=0.344)$, number of laminec- 
Table 2.

\begin{tabular}{lccc}
\hline & Epidural hematoma & Control & $P$-value \\
\hline No. of patients & 4 & 133 & \\
Age & $70.5 \pm 11.0$ & $72.5 \pm 9.5$ & 0.672 \\
Disease duration & $17.3 \pm 15.6$ & $33.1 \pm 0.2$ & 0.344 \\
Gender (No. [\%] of Males) & $4(100)$ & $64(48.1)$ & 0.041 \\
No. of laminectomy & $1 \pm 0$ & $1.4 \pm 0.6$ & 0.165 \\
Levels decompressed & $1.75 \pm 0.5$ & $3.7 \pm 0.7$ & $3.1 \times 10^{-7}$ \\
Blood loss & $54 \pm 27.3$ & $68.2 \pm 55.0$ & 0.609 \\
Time of surgery & $129.5 \pm 75.8$ & $88.1 \pm 40.7$ & 0.053 \\
Drain output & $113.5 \pm 115.2$ & $234.6 \pm 119.4$ & 0.048 \\
No. (\%) of Coagulopathy & $1(25)$ & $12(9)$ & 0.131 \\
No. $\%$ ) of Dural tear & $1(25)$ & $8(6)$ & 0.284 \\
\hline
\end{tabular}

Table 3.

\begin{tabular}{lcc}
\hline & $\chi^{2}$ & $P$-value \\
\hline Age & 3.472 & 0.0624 \\
Gender & $4.5 \times 10^{-8}$ & 0.999 \\
Multilevel procedures & $5.2 \times 10^{-9}$ & 0.999 \\
Levels decompressed & 15.06 & 0.0001 \\
Blood loss & $4.3 \times 10^{-7}$ & 0.999 \\
Drain output & 4.577 & 0.0324 \\
Coagulopathy & $8 \times 10^{-9}$ & 0.999 \\
Dural tear & $2.6 \times 10^{-6}$ & 0.998 \\
\hline
\end{tabular}

tomies $(1,1.4, \mathrm{p}=0.165)$, levels decompressed $(1.75,3.7, \mathrm{p}$ $\left.=3.1 \times 10^{-7}\right)$, blood loss in $\mathrm{mL}(54,68.2, \mathrm{p}=0.609)$, time of surgery in minutes $(129.5,88.1, \mathrm{p}=0.053)$, drain output in $\mathrm{mL}(113.5,234.6, \mathrm{p}=0.048)$, coagulopathy $(1 \%-25 \%$, $12 \%-9 \%, \mathrm{p}=0.131)$, and dural tear $(1 \%-25 \%, 8 \%-6 \%, \mathrm{p}=$ 0.284) (Table 2). The SPSL group differed significantly from the control group in that there were more men, the levels decompressed were significantly higher, and the drain outputs were significantly lower $(\mathrm{p}<0.05)$.

From our logistic regression analysis, the levels of decompression were significantly higher $\left(\chi^{2}=15, \mathrm{p}=0.0001\right)$ and the drain outputs were smaller $\left(\chi^{2}=4.6, \mathrm{p}=0.03\right)$ in the hematoma group (Table 3).

\section{Case presentation}

The case is that of a 72-year-old man with a medical history of diabetes and taking coagulopathy medication (Apixaban) who presented with spinal stenosis at levels L2-3 (Table 1; Nb. 3, Fig. 1A). He complained of bilateral thigh pain and intermittent claudication at 200 meters without weakness and urinary disorder. His preoperative JOA score was 20/29 (normal score, 29 points); the visual analogue scale (VAS; from 0 [no pain] to 100 [extreme amount of pain]) for low back pain was 30; the VAS score for leg pain was 80. L2-3 SPSL was performed (Fig. 1B). On postoperative day 2 , bilateral drop foot was noted. The drain output was only $27 \mathrm{~mL}$. The nerve findings, elicited using the manual muscle test, were as follows (right side/left side): iliotibialis, $5 / 5$; quadriceps, 5-/4; tibialis anterior, $3 / 3$; and extensor hal- lux longus, 3/3. Magnetic resonance imaging was obtained emergently, showing epidural hematoma ascending to the L1 level (Fig. 1C, D). Emergency hematoma evacuation was performed thereafter, with L1 laminectomy added to remove hematoma stretching from L1 to L2. Lower extremity weakness and leg pain completely recovered 1 month following surgery.

\section{Discussion}

In the present study, there were four patients $(3.7 \%)$ who had occurrence of spinal epidural hematoma after SPSL. The risk factors for spinal epidural hematoma included single-level laminectomy higher than the L2-3 level and reduced drain output. Regarding incidence and risk factors for epidural hematoma following spinal surgery, Kao et al. reported a frequency of $0.16 \%(25 / 15,562)^{7}$. Increased diastolic blood pressure, the use of gelfoam, and increased postoperative drain output led to increased risk ${ }^{7}$. Awad et al. reported a risk of epidural hematoma of $0.21 \%(32 / 14,932)$, demonstrating that the risk factors included use of NSAIDs (Non-Steroidal Anti-Inflammatory Drugs), Rh-positive blood, age $>60$ years, and surgery involving six or more levels $^{8}$. Kou et al. reported a risk of $0.1 \%(12 / 12,000)$, demonstrating that the risk factors included multilevel procedures and preoperative coagulopathy ${ }^{9}$. Amiri et al. reported a $0.22 \%(10 / 4,568)$ risk of spinal epidural hematoma, demonstrating that the risk factors included increased alcohol consumption, revision surgery, and multilevel surgery ${ }^{10)}$. Aono et al. reported a risk spinal epidural hematoma of $0.41 \%(\mathrm{n}=6,356)^{11}$. In the aforementioned studies, the prevalence of epidural hematoma ranged from $0.1 \%$ to $0.4 \%^{12}$. For SPSL postoperative hematoma, Baghdadi et al. reported a $2.7 \%$ risk $(1 / 37)^{13)}$. The frequency in our study was $3.7 \%$ (4/137). This prevalence of SPSL postoperative hematoma ranged from $2.7 \%$ to $3.7 \%$ is about 10 times as frequent as reports ${ }^{12}$ from conventional spine surgery. In the present study concerning SPSL, single-level decompression above L2-3 and reduced drain output were risk factors for spinal epidural hematoma. At these higher levels, the effect of hematoma may be more pronounced as there is reduced 

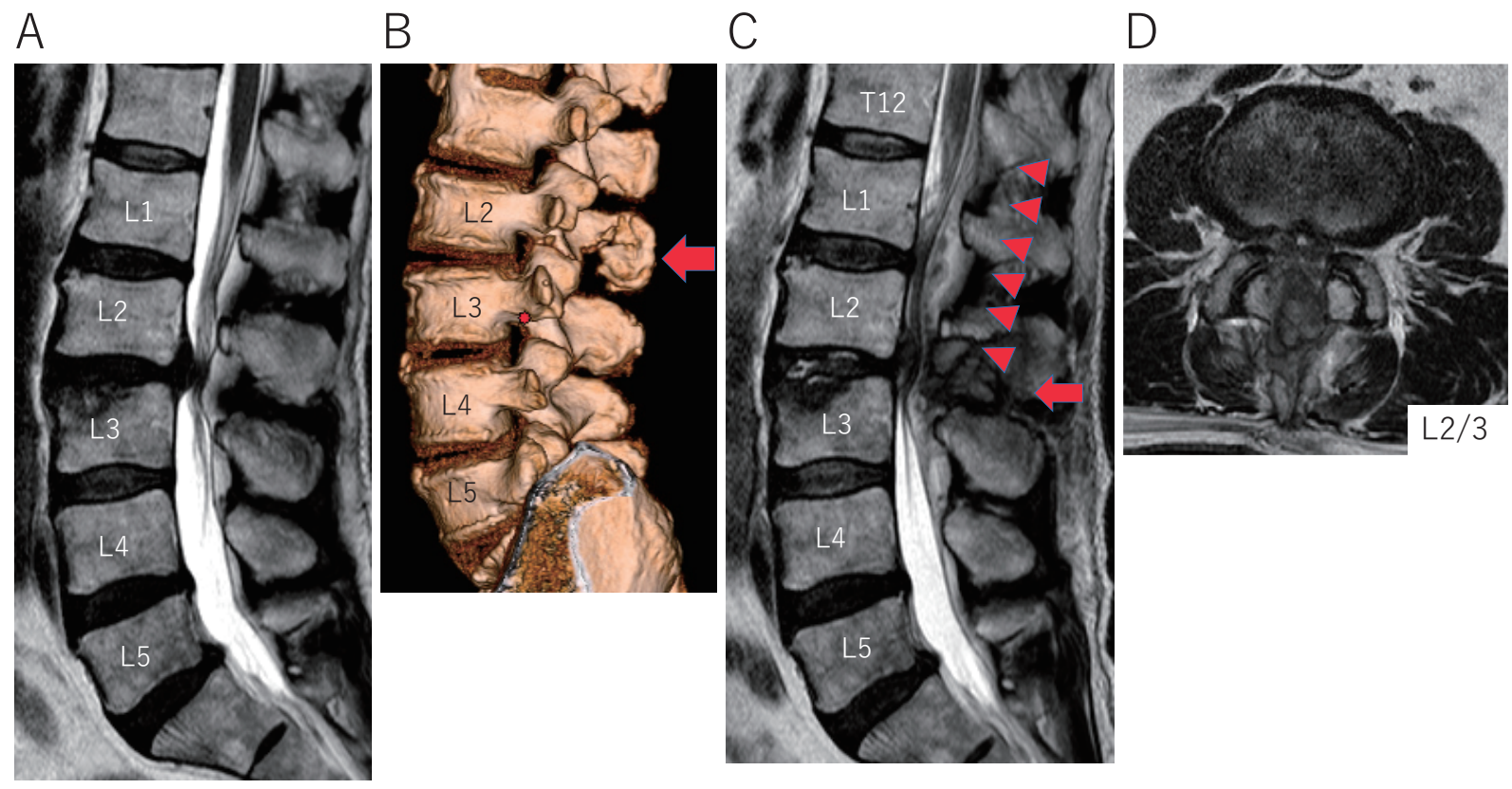

Figure 1. A 72-year-old man before and after a lumbar L2-3 spinous process-splitting laminectomy: Magnetic resonance imaging (A, C, D-C) and CT (B). (A) Preoperative T2-weighted sagittal MRI showing L2-3 spinal stenosis; (B) Postoperative 3-D CT reconstruction of the lumbar spine; arrow at the level of L2-3 decompression; (C) Postoperative T2weighted sagittal MRI showing epidural hematoma (arrow head) ascending to the level of L1; (D) T2-weighted axial MRI showing the L2-3 level in the setting of postoperative paralysis.

canal width, and drain failure may also occur with this limited space. Based on this, we would urge caution when performing this procedure at the level of L2-3 or above. One limitation of our study is that the number of cases is small, requiring confirmation of our findings in a larger population.

\section{Conclusion}

We investigated the risk of postoperative epidural hematoma in SPSL. Epidural hematoma occurred in 4 out of 137 cases, with a frequency of $3.7 \%$. Single-level decompression above L2-3 and reduced drain output were risk factors. As this technique entails less decompression compared with conventional methods, special attention must be paid to levels superior to L2-3 with narrower canal and potentially poorer drainage potential.

Disclaimer: Sumihisa Orita is one of the Editors of Spine Surgery and Related Research and on the journal's Editorial Committee. He was not involved in the editorial evaluation or decision to accept this article for publication at all.

Conflicts of Interest: The authors declare that there are no relevant conflicts of interest.

Author Contributions: Y. Eguchi: project development, data collection, manuscript writing; M. Suzuki: support of surgical treatment; T. Sato: data collection; H. Yamanaka: data collection; H. Tamai: data collection; T. Kobayashi: data collection; S. Orita: data collection; M. Suzuki: data collection; K. Inage: project development; H. Kanamoto: data collection; K. Abe: data collection; M. Norimoto: project development, data collection; T. Umimura: data collection; Y. Aoki: data collection; M. Koda: data collection; T. Furuya: data collection; J. Nakamura: data collection; T. Akazawa: data collection; K. Takahashi: scientific guarantor of this manuscript; S. Ohtori: scientific guarantor of this manuscript

Ethics and Consent to Participate: We declare that all protocols involving humans have been approved by the Shimoshizu National Hospital and have been performed in accordance with the ethical standards laid down in the 1964 Declaration of Helsinki and its later amendments. We declare that all participants provided written informed consent before their inclusion in this study.

\section{References}

1. Ishimoto Y, Yoshimura N, Muraki S, et al. Associations between radiographic lumbar spinal stenosis and clinical symptoms in the general population: the Wakayama spine study. Osteoarthritis Cartilage. 2013;21(6):783-8.

2. Weiner BK, Fraser RD, Peterson M. Spinous process osteotomies to facilitate lumbar decompressive surgery. Spine (Phila Pa 1976). 1999;24(1):62-6.

3. Weiner BK, Walker M, Brower RS, et al. Microdecompression for lumbar spinal canal stenosis. Spine (Phila Pa 1976). 1999;24(21): 2268-72.

4. Nakai O, Ookawa A, Yamaura I. Long-term roentgenographic and functional changes in patients who were treated with wide fenestration for central lumbar stenosis. J Bone Joint Surg Am. 1991;73 (8):1184-91.

5. Poletti CE. Central lumbar stenosis caused by ligamentum flavum: 
unilateral laminotomy for bilateral ligamentectomy: preliminary report of two cases. Neurosurgery. 1995;37(2):343-7.

6. Watanabe K, Hosoya $T$, Shiraishi $T$, et al. Lumbar spinous process-splitting laminectomy for lumbar canal stenosis. Technical note. J Neurosurg Spine. 2005;3(5):405-8.

7. Kao FC, Tsai TT, Chen LH, et al. Symptomatic epidural hematoma after lumbar decompression surgery. Eur Spine J. 2015;24 (2):348-57.

8. Awad JN, Kebaish KM, Donigan J, et al. Analysis of the risk factors for the development of post-operative spinal epidural haematoma. J Bone Joint Surg Br. 2005;87(9):1248-52.

9. Kou J, Fischgrund J, Biddinger A, et al. Risk factors for spinal epidural hematoma after spinal surgery. Spine. 2002;27(15):167073.

10. Aono H, Ohwada T, Hosono N, et al. Incidence of postoperative symptomatic epidural hematoma in spinal decompression surgery. J Neurosurg Spine. 2011;15: 202-5.
11. Amiri AR, Fouyas IP, Cro S, et al. Postoperative spinal epidural hematoma (SEH): incidence, risk factors, onset, and management. Spine J. 2013;13(2):134-40.

12. Schroeder GD, Kurd MF, Kepler CK, et al. Postoperative Epidural Hematomas in the Lumbar Spine. J Spinal Disord Tech. 2015;28 (9):313-8.

13. Baghdadi YM, Moussallem CD, Shuaib MA, et al. Lumbar spinous process-splitting laminoplasty: A novel technique for minimally invasive lumbar decompression. Orthopedics. 2016;39(5): e950-6.

Spine Surgery and Related Research is an Open Access journal distributed under the Creative Commons Attribution-NonCommercial-NoDerivatives 4.0 International License. To view the details of this license, please visit (https://creativeco mmons.org/licenses/by-nc-nd/4.0/). 